\title{
Cavity Spintronics Gets More with Less
}

\section{A new design for a cavity spintronic device obtains a strong photon-magnon coupling with a magnet that is over 1000 times smaller than previously used magnets.}

\section{by Can-Ming Hu*}

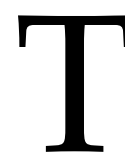
he two physics pioneers Richard Feynman and Philip Anderson envisioned different paths for future discoveries. "There's plenty of room at the bottom," said Feynman, believing that new physics lay at ever smaller scales. By contrast, Anderson said "more is different" [1], implying that as systems grow larger and become complex, new collective phenomena will emerge. These paths are usually divergent, but two novel designs for hybrid photon-magnet cavities show how to get large-scale effects from a smaller sized system. The so-called cavity spintronic devices - independently developed by Justin Hou and Luqiao Liu at the Massachusetts Institute of Technology,

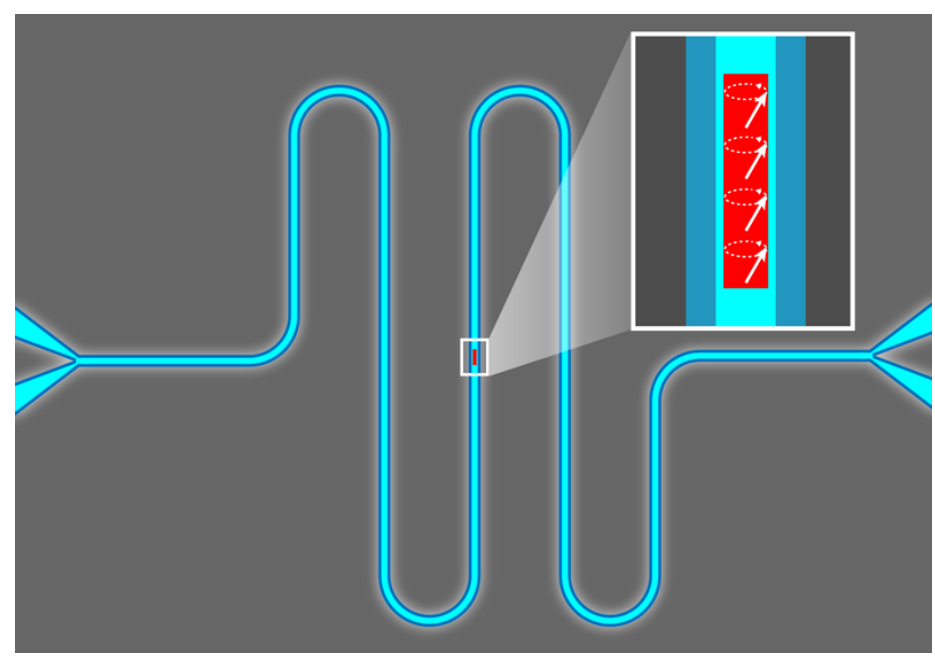

Figure 1: In the new cavity spintronic designs [2, 3], a small magnetic stripe (red) is placed in the center of a microwave cavity etched onto a silicon substrate. The design creates a strong coupling between photons in the cavity and spin excitations in the magnet. A representation of such excitations is shown in the inset. (APS/Alan Stonebraker)

\footnotetext{
*Department of Physics and Astronomy, University of Manitoba, Winnipeg, MB, Canada
}

Cambridge [2], and Yi Li from Oakland University, Michigan, and colleagues [3] — consist of a microwave cavity and a small magnetic stripe, allowing photons and spins to interact with each other. The magnetic component here is much smaller than that used in previous cavity spintronic devices, and yet the two teams are able to engineer a strong coupling between the cavity's photons and the stripe's spins. The reduction in the magnet's size opens up the possibility of placing cavity spintronic devices on a chip to convert between magnetically stored information and light signals.

Cavity spintronics (also known as spin cavitronics and cavity magnonics) emerged in 2010 out of Andersoninspired theoretical work on the interaction between nanomagnets and microwave cavities [4, 5]. Normally, the rate of dipole interactions between photons in a cavity and a single spin is very small, as given by the vacuum Rabi frequency $g_{0}$. However, a collective excitation of spins, called a magnon, can have a much stronger coupling to a photon-with the interaction rate exceeding both the photon and magnon dissipative rates [6]. The key idea stems from the theory of cooperative dynamics [7]: Since the $N$ spins in a ferromagnet couple coherently with the photon via the magnon excitation, the magnon-photon coupling rate $g$ has a $\sqrt{N}$-fold enhancement over the single-spin coupling rate: $g=g_{0} \sqrt{N}$. Cavity spintronic devices have demonstrated enhanced coupling rates, paving the way for magnon-based transducers that can connect different parts of a hybrid quantum system. Such transducers have attracted broad interest from communities in various fields, such as quantum information, quantum optics, spintronics, cavity optomechanics, and light-matter interaction [8].

Although a lot of progress has been made in cavity spintronics, the reliance on large numbers of spins to enhance the coupling rates has limited its applications. Nearly all cavity spintronics experiments to date have used yttrium iron garnet (YIG) in the form of millimeter-scale components containing $N \sim 10^{16}-10^{19}$ spins. YIG is a ferromagnetic insulator with a low magnon dissipative rate, but it is very difficult to fabricate at small dimensions, as well as to integrate with other devices on electronic chips. The field of cavity spintronics will need a bit of Feynman's miniaturization if it is to become practical in future nanotechnologies. 
The new cavity spintronic devices from Hou et al. [2] and $\mathrm{Li}$ et al. [3] are a step in the nanoscale direction. Both groups replace the bulky YIG elements with thin-film stripes of permalloy, a nickel-iron alloy with a larger spin density than YIG. And yet the small size of the micrometer-wide stripes means that they have 1000 times less spins than in the smallest YIG magnets that were previously used. So how do the research teams obtain strong magnon-photon coupling? They do so by carefully constructing the photon cavities. Both teams' cavities are planar superconducting resonators that look like curvy racetracks on a silicon substrate (Fig. 1). The permalloy stripes are placed in the center of the racetrack, and an external magnetic field is used to set the magnon mode frequency near the cavity resonance frequency. The device geometry has two important effects: it reduces the volume of the cavity mode, and it ensures that the magnetic-field component of the cavity field overlaps strongly with the magnon mode. Both these effects contribute to boosting the coupling rate [9], as demonstrated in a recent work with YIG films [10].

In their experiment, $\mathrm{Li}$ et al. achieve a coupling rate of $152 \mathrm{MHz}$ using a permalloy stripe with dimensions of $900 \mu \mathrm{m} \times 14 \mu \mathrm{m} \times 30 \mathrm{~nm}$ [3], while Hou and Liu report a $171-\mathrm{MHz}$ rate in a stripe of size $2000 \mu \mathrm{m} \times 8 \mu \mathrm{m} \times 50 \mathrm{~nm}$ [2]. The couplings from the stripes are as strong as those achieved with YIG magnets, despite the stripes having only $N \sim 10^{13}$ spins. Both teams explored other ways to further enhance the coupling: Li et al. studied the nonlinear regime of their superconducting resonator; Hou and Liu investigated complex cavity designs with reduced impedance. Since the teams use metallic ferromagnets with conventional silicon substrates, their new devices can be easily integrated with either silicon-based or superconducting quantum circuits. The results therefore mark a critical step towards future magnon-based hybrid systems for on-chip scalable quantum information processing.

If the magnets in cavity spintronics can be further reduced in size to the nanoscale, other opportunities open up. Nanomagnets are often used in magnetic tunnel junctions (MTJs), which work as spin-torque nano-oscillators for generating continuous-wave microwave signals [11]. By exploiting the cavity spintronics approach, an array of MTJs could be assembled and synchronized through a coupling to a single microwave cavity mode. Through this cavity-mediated synchronization, a macroscopic ensemble of many nanooscillators could become a powerful microwave source. Here again, Feynman and Anderson's visions would unite to produce new possibilities.

This research is published in Physical Review Letters.

\section{REFERENCES}

[1] P. W. Anderson, "More is different," Science 177, 393 (1972).

[2] J. T. Hou and L. Liu, "Strong coupling between microwave photons and nanomagnet magnons," Phys. Rev. Lett. 123, 107702 (2019).

[3] Y. Li et al., "Strong coupling between magnons and microwave photons in on-chip ferromagnet-superconductor thin-film devices," Phys. Rev. Lett. 123, 107701 (2019).

[4] Ö. O. Soykal and M. E. Flatté, "Strong field interactions between a nanomagnet and a photonic cavity," Phys. Rev. Lett. 104, 077202 (2010).

[5] C.-M. Hu, "Dawn of cavity spintronics," Physics in Canada 72 , No. 2, 76 (2016).

[6] H. Huebl, C. W. Zollitsch, J. Lotze, F. Hocke, M. Greifenstein, A. Marx, R. Gross, and S. T. B. Goennenwein, "High cooperativity in coupled microwave resonator ferrimagnetic insulator hybrids," Phys. Rev. Lett. 111, 127003 (2013).

[7] R. H. Dicke, "Coherence in spontaneous radiation processes," Phys. Rev. 93, 99 (1954).

[8] D. Lachance-Quirion, Y. Tabuchi, A. Gloppe, K. Usami, and Y. Nakamura, "Hybrid quantum systems based on magnonics," Appl. Phys. Express 12, 070101 (2019).

[9] X. Zhang, C.-L. Zou, L. Jiang, and H. X. Tang, "Strongly coupled magnons and cavity microwave photons," Phys. Rev. Lett. 113, 156401 (2014).

[10] L. McKenzie-Sell, J. Xie, C.-M. Lee, J. W. A. Robinson, C. Ciccarelli, and J. A. Haigh, "Low-impedance superconducting microwave resonators for strong coupling to small magnetic mode volumes," Phys. Rev. B 99, 140414 (2019).

[11] S. Kaka, M. R. Pufall, W. H. Rippard, T. J. Silva, S. E. Russek, and J. A. Katine, "Mutual phase-locking of microwave spin torque nano-oscillators," Nature 437, 389 (2005).

10.1103/Physics. 12.97 